\title{
Redox-responsive tumor targeted dual-drug loaded biocompatible metal-organic frameworks nanoparticles for enhancing anticancer effects
}

\author{
Chang Liu' ${ }^{1}$, Xiaoyu Xu' ${ }^{1}$, Junnian Zhou ${ }^{1,2}$, Jiaqi Yan ${ }^{1,3}$, Dongqing Wang ${ }^{4}$ and Hongbo Zhang ${ }^{1,4^{*}}$ (D)
}

\begin{abstract}
Metal-organic frameworks (MOFs) have proven to be a promising class of drug carriers due to their high porosity, crystalline properties with defined structure information, and abundant surface chemistry for further functionalization. However, there has not been extensive research on MOF-based drug carriers with stimuli-responsive, dual-drug delivery, and tumor targeting functions. Here, we demonstrate the strategy of constructing a redox responsive and tumor-targeted MOF as dual-drug carrier by anchoring functional disulfide anhydride and folic acid molecules to the organic links of MOFs, respectively. The MOF composites show the controlled release of loaded 5-fluorouracil (5-FU) entrapped within UiO-66- $\mathrm{NH}_{2}$ nanostructures modified with dichloroacetic acid, which acts as a synergistical drug to 5-FU in cancer cells. In addition, the overexpressed GSH in cancer cells attacks the thiolate moiety and is oxidized in the process as it cleaves the disulfide bonds, thereby achieving redox stimuli-responsive drugs release in MOFs. The confocal laser scanning microscopy further proved that conjugation of folic acid to the MOF surface can significantly enhance the targeting uptake of cancer cells. This work paves the way to the construction of stimuli responsive tumor-targeted Nano MOF based drug carriers with potential for cancer therapies.
\end{abstract}

Keywords: Metal-organic frameworks, Nanomedicine, Redox-responsive, Dual-drug delivery, Target therapy

\section{Introduction}

The increasing incidence and mortality of cancer over the past few decades has challenged current drug treatments. Many approaches are limited by their non-specific distribution throughout the body, resulting in needing of high amount of drug doses, rapid drug clearance, poor pharmacokinetics, and severe adverse drug effects. Although many different strategies have been developed to improve the efficacy of conventional drugs, nanoparticle-based drug delivery systems have received increasing attention

\footnotetext{
*Correspondence: hongbo.zhang@abo.fi

1 Pharmaceutical Sciences Laboratory and Turku Bioscience Center, Åbo

Akademi University, 20520 Turku, Finland

Full list of author information is available at the end of the article
}

over the past 20 years. By using these novel nanosystems, many of the shortcomings of small molecule drugs can be effectively alleviated [1]. Generally, nanomedicines are composed of active pharmaceutical ingredients incorporated into nanoparticle carriers. Various nanocarriers such as polymer nanoparticles [2, 3], micelles [4], liposomes [5], iron oxides [6], and gold [7] have been developed and used for this purpose. The good nanocarriers have key properties including small and tunable size, high drug loading capacity and efficiency, favorable surface properties, in demand drug release kinetics, improved pharmacokinetics, and biocompatibility. Besides, nanoparticles can specifically target diseased tissues (such as tumor regions) by binding to target receptors [8], and can be engineered to enable theranostics [9], 
while are difficult to be achieved with conventional therapies. Another important part of nano-drug delivery is the stimuli responsive mechanism [10], which determines whether the drug delivery system has a controlled release function. The precise design of the controlled release behavior should be based on a thorough understanding of the relevant stimulation signals and release mechanisms in physiological and pathological conditions. Nanomedicines that respond to $\mathrm{pH}[11,12]$, temperature [13], light [14], redox state [15], magnetic fields [16, 17], biomolecules [18], or combinations thereof have been developed and proved. The clinical success of nanoparticle-based therapy illustrates the power of this approach. Currently, many promising nanoparticles-drug combinations are at different stages of clinical development.

Metal-organic frameworks (MOFs), as a new type of porous and crystalline materials, assembled from metal ions (or metal clusters) and organic linkers, have shown various potential applications, such as adsorption of gas or water [19], catalysis [20], sensing [21], proton conduction [22], and drug delivery [23, 24]. In the past few decades, nanoscale metal-organic frameworks have attracted great attention in the field of particle-mediated cancer drug delivery [25]. They have shown advantages over conventional drug carrier materials, for example, they have various morphologies with well-defined crystal structure, high surface area, large drug loading capacity, and intrinsic biodegradability caused by relatively unstable coordination bonds. Due to its hybrid nature related to organic ligands and inorganic building blocks, MOFs have tremendous opportunities for further customization and functionalization. In general, the organic components of MOFs are more concerned with the subsequent post-synthetic modification (PSM) [26], and organic molecules are covalently connected through various organic reactions, thereof providing new chemical functions for grafting of drug molecules. Through reasonable PSM, MOFs drug carriers can co-deliver two different chemical anticancer drugs and potentially stimuli-responsive release drugs to tumor cells because cancer cells have higher overexpressing reducing components than normal cells [27]. The fabrication of stimuli-responsive surfaces on MOFs for environmentally sensitive site-specific drug delivery can also reduce the risk of premature drug leakage. Although, there are reports that some targeted MOF carrier could delivery two different drugs and achieve targeting release around cancer cells due to the lower $\mathrm{pH}$ than normal cells, the MOF-based drug delivery system with stimuli-responsive and targeting functions has not been explored. In addition to introducing stimuli-responsive/targeting MOF hybrids as a new functional material for targeting tumor and controlled drug release, the use of MOFs also has important advantages over other stimuli-responsive porous carriers. Furthermore, the formation of the MOFs determines a uniform pore size, and therefore, it is expected that the released loads will reveal a more controlled release profile. The conjugation of anhydride acids with MOFs as stimuli-responsive capping units is particularly interesting because biological components can be used to unlock the chemical bonds of caps to achieve rapid drug release. Here, folic acid (FA) is one of the most commonly used tumor-targeting ligands in drug delivery system because folic acid receptors are well-known tumor markers and the conjugation chemistry to therapeutic agents is well characterized [28].

In this study, we report the design of redox-responsive dual-drug delivery tumor-targeted MOFs nanocarriers (Scheme 1). Here, the zirconium MOF UiO-66- $\mathrm{NH}_{2}(\mathrm{UiO}$ stands for Universitetet i Oslo) was selected as the model as a drug carrier [29]. During the synthesis of nanoparticles, we introduced dichloroacetic acid (DCA) as part of the organic links fused in the framework to control the size and as a second anticancer drug to enhance the cancer cell cytotoxicity of the dual-drug delivery system. The amino groups on the surface of the nanoparticles were then modified with the 3,3'-dithiodipropionic acid anhydride (DTDPA) to link with tumor-targeted components. After entrapping 5-fluorouracil (5-FU) in the DTDPAmodified MOFs, the resulting nanoparticles were then coordinated by aminated folic acid molecules to prevent drug leakage. The nanocarriers then accumulated in tumor tissues through the EPR and FA targeting effects and uptaken in cancer cells via endocytosis. Thereafter, the redox responsive disulfide bonds were broken down in the presence of overexpressed GSH. The structure of nanocarriers, on the other hand, deconstructed with the release of contained drugs, enhancing anticancer effects by synergistic of DCA and 5-FU.

\section{Results and discussion}

Dichloroacetic acid (DCA) is a pyruvate D-kinase inhibitor that is overexpressed in cancer cells. Its cytotoxic effect on cancer cells depends on effective cytoplasmic release and mitochondrial localization, making it an ideal probe molecule for cellular uptake mechanism [30]. In order to promote cytoplasmic release through passive diffusion, UiO-66- $\mathrm{NH}_{2}$ (DCA-UiO-66- $\mathrm{NH}_{2}$ ) doped with dichloroacetic acid (DCA) was synthesized by heating $\mathrm{N}, \mathrm{N}^{\prime}$-dimethylformamide (DMF) solution in the oven at $120{ }^{\circ} \mathrm{C}$ for $24 \mathrm{~h}$, containing $\mathrm{Zr}^{4+}$, 2-amino-1,4-benzenedicarboxylicacid $\left(\mathrm{NH}_{2}-\mathrm{H}_{2} \mathrm{BDC}\right)$ ligands, and dichloroacetic acid, respectively, with gently stirring. In the synthesis of the DCA-UiO-66- $\mathrm{NH}_{2}$, different ratios of metal ions and ligands were used to obtain the optimal nanoscale products. As a regulator of $\mathrm{UiO}-66-\mathrm{NH}_{2}$ solvothermal synthesis, it showed that DCA was incorporated into the 


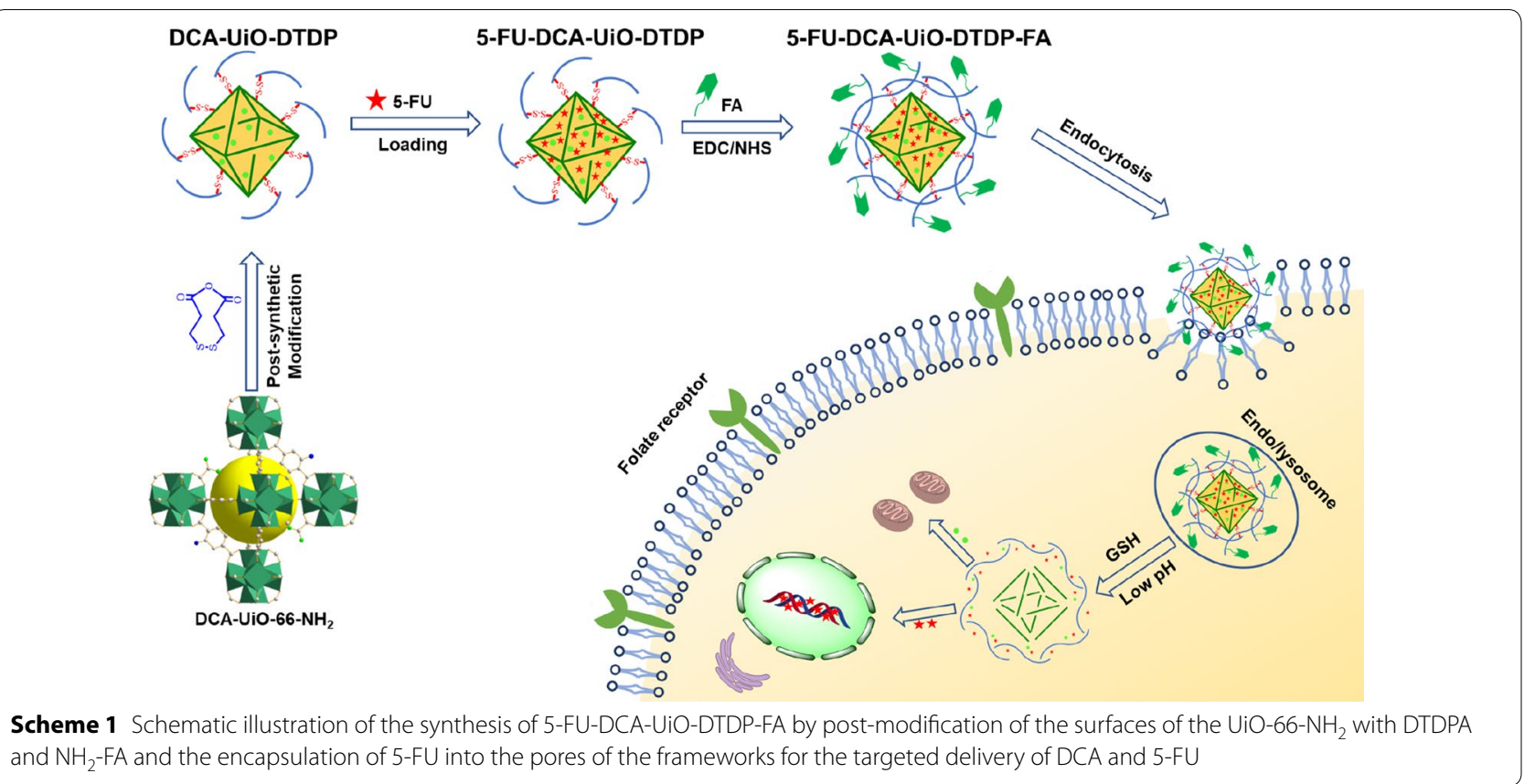

defects of UiO-66- $\mathrm{NH}_{2}$ nanoparticles and their surfaces, as shown in Additional file 1: Fig. S1. For DCA-UiO66- $\mathrm{NH}_{2}$, when the molar ratio of $\mathrm{Zr}^{4+}, \mathrm{NH}_{2}-\mathrm{H}_{2} \mathrm{BDC}$, and DCA was 1:1:9, the particle size of the obtained product was around $50 \mathrm{~nm}$, as characterized by transmission electron microscopy (TEM) (see Additional file 1: Fig. S1a), which is superior to most MOF-based targeted drug carriers reported in the literature. In order to prove the surface chemistry and structure of the synthesized DCAUiO-66- $\mathrm{NH}_{2}$, Fourier-transform infrared spectroscopy (FT-IR) and powder X-ray diffraction (PXRD) analysis were performed. The FT-IR spectra of DCA-UiO-66- $\mathrm{NH}_{2}$ showed a new band in the carboxylic acid region but shifted compared to free DCA, which was characteristic of the carboxylic acid in DCA that connected to the $\mathrm{Zr}_{6}$ units. The presence of a new band related to the $\mathrm{C}-\mathrm{Cl}$ stretching at around $800 \mathrm{~cm}^{-1}$ was appreciable, and no shifting was observed (Additional file 1: Fig. S2). Furthermore, their PXRD patterns closely matched the simulated patterns derived from the single-crystal X-ray diffraction data, which proved the successful synthesis of the DCAUiO-66- $\mathrm{NH}_{2}$ (Fig. 2).

The covalent post-synthetic modification of DCAUiO-66- $\mathrm{NH}_{2}$ was explored by using an alkyl anhydride to produce carboxylic group terminal frameworks designated DCA-UiO-DTDP (in Additional file 1: Fig. S3). According to the previous literature in Additional file 1: Fig. S4, 3,3'-dithiodipropionic acid anhydride (DTDPA) was obtained by acylation of 3,3'-dithiodipropionic (DTDP) acid with acetyl chloride [31]. The resonance at $\delta=12.35 \mathrm{ppm}$ corresponded to the carboxyl proton of the DTDP, and the peak disappeared after refluxing in acetyl chloride, which indicated the successful formation of DTDPA in ${ }^{1} \mathrm{H}$ NMR in Additional file 1: Fig. S5. In a typical post-synthetic modification reaction, after the synthesis of DCA-UiO-66- $\mathrm{NH}_{2}, 1: 1$ molar ratio of DTDPA was placed in the above DMF solution. After standing at room temperature for $24 \mathrm{~h}$, the sample was rinsed with methanol to extract byproducts from the porous solids. The modification was confirmed by nuclear magnetic resonance (NMR) spectroscopy. The sample of post-synthetic modification treated DCA-UiO-66- $\mathrm{NH}_{2}$ materials was digested with $\mathrm{D}_{2} \mathrm{SO}_{4}$ and DMSO- $d_{6}$ for examination by ${ }^{1} \mathrm{H}$ NMR spectroscopy. The digestion of unmodified DCA-UiO-66- $\mathrm{NH}_{2}$ mainly showed resonances related to 2-amino-1,4-benzene dicarboxylic acid, and the resonance at $\delta=6.2 \mathrm{ppm}$ corresponded to the $-\mathrm{CCl}_{2} \mathrm{H}$ proton of DCA in Additional file 1: Fig. S6. It is not possible to quantitatively determine the DCA loading value from the NMR spectra alone. However, according to the external standard method of ${ }^{1} \mathrm{H}$ NMR standard curve of DCA in Additional file 1: Fig. S6, from the ${ }^{1} \mathrm{H}$ NMR spectra of acid digested DCA-UiO-66- $\mathrm{NH}_{2}$ samples, it was estimated that the incorporation amount of DCA was $17.96 \%$. At the same time, some minor impurities were also observed in the aromatic region of DCA-UiO-DTDP, which seemed to be associated with the amino-functionalized benzenedicarboxylate (BDC) ligand (Additional file 1: Fig. S7). The TEM in Fig. 1b and XRD in Fig. 2 confirmed that there was no significant change in the 

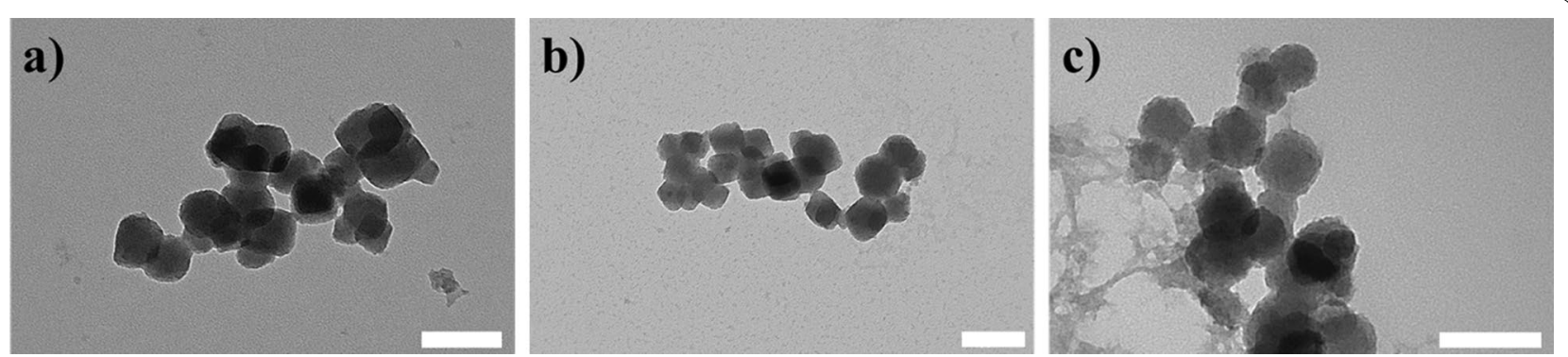

Fig. 1 TEM images of a as-synthesized DCA-UiO-66-NH ${ }_{2}$, b DTDPA-functionalized DCA-UiO-66- $\mathrm{NH}_{2}$ (DCA-UiO-DTDP), and $\mathbf{c} \mathrm{NH}_{2}-\mathrm{FA}$ modified DCA-UiO-DTDP (DCA-UiO-DTDP-FA) nanoparticles, the scale bars are $100 \mathrm{~nm}$

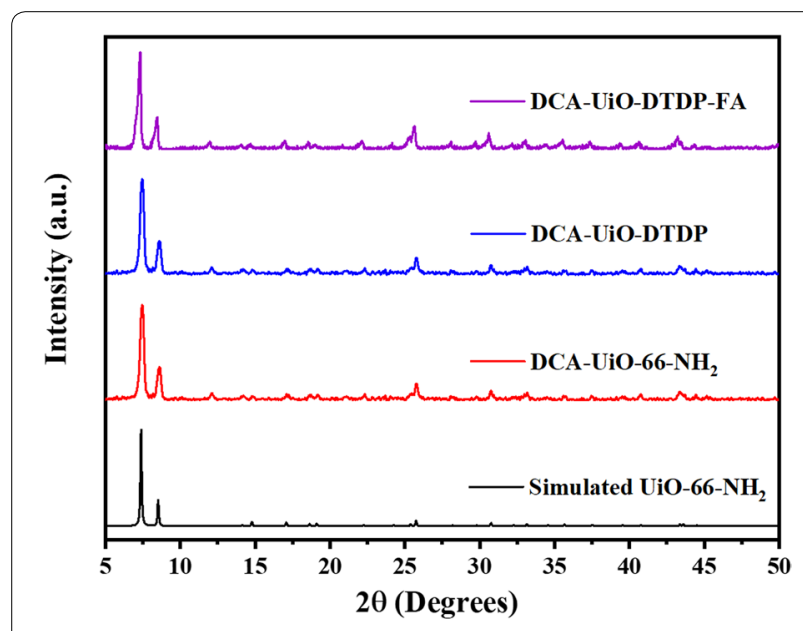

Fig. 2 Stacked PXRD patterns of DCA-UiO-66-NH 2 , DCA-UiO-DTDP and DCA-UiO-DTDP-FA, compared to the simulated pattern of $\mathrm{UiO}-66-\mathrm{NH}_{2}$

morphology and crystallinity of DCA-UiO-DTDP nanoparticles after modification with anhydrate substance.

Next, we tried to establish an active targeting model on the MOFs platform by performing post-synthetic modification on the modified surfaces of DCA-UiODTDP. Since there are available binding sites on the surface of DCA-UiO-DTDP, the terminal amino group of aminated folic acid $\left(\mathrm{NH}_{2}-\mathrm{FA}\right)$ has a great chance to be coordinated with the ligands on the surface of DCAUiO-DTDP. Additional file 1: Fig. S8 shows the synthesis procedure of aminated folic acid between folic acid and ethylenediamine [32]. The obtained $\mathrm{NH}_{2}$-FA was analyzed in Additional file 1: Fig. S9 by using ${ }^{1} \mathrm{H}$ $\mathrm{NMR}$. The $\mathrm{NH}_{2}$-FA-modified DCA-UiO-DTDP was prepared by stirring a solution containing DCA-UiODTDP and aminated folic acid at room temperature for $6 \mathrm{~h}$. The comparison of the FT-IR spectra of DCAUiO-DTDP and DCA-UiO-DTDP-FA showed that the absorption peaks of FA had a strong contribution to the FT-IR spectra of DCA-UiO-DTDP-FA (Additional file 1: Fig. S10), which indicated that the reactions were successful. In contrast, the PXRD patterns of the DCAUiO-DTDP-FA and its parent MOF did not show any differences, demonstrating the integrity of their frameworks after the reaction (Fig. 2). After the anchoring with the $\mathrm{NH}_{2}-\mathrm{FA}$, a thin and uniform layer was formed on the surface of DCA-UiO-66-DTDP crystals, and its thickness was about several nanometers, as shown in Fig. 1c.

For biological applications, the biocompatibility and cytotoxicity of the anticancer drugs and prepared materials must be evaluated, and here we have used the cell proliferation assay Roche diagnostics reagent (WST1). The cytotoxicity of the DCA-UiO-DTDP, DCAUiO-DTDP-FA and free DCA (in the form of sodium dichloroacetate, $\mathrm{NaDCA}$, eliminating cytotoxic effects caused by $\mathrm{pH}$ changes) were measured towards breast cancer cell line MDA-MB-231 and non-tumorigenic epithelial cell line MCF-10A. The WST-1 assay results for NaDCA are shown in Additional file 1: Fig. S11, confirming that DCA has little effect on cell proliferation due to its natural hydrophilic properties leading to poor internalization, and a decrease in cell viability was observed only at concentrations higher than $2 \mathrm{mg} / \mathrm{mL}$, with $69.5 \pm 9.9 \%$ and $91.0 \pm 12.6 \%$ viabilities after incubation of NaDCA in MDA-MB-231 and MCF-10A, respectively. Then, the cell viability was measured by the WST-1 assay for $72 \mathrm{~h}$ with different concentrations of the DCA-UiO-DTDP and DCAUiO-DTDP-FA $(10,20,50,100,200,500$, and $1000 \mu \mathrm{g} /$ $\mathrm{mL}$ ). As can be seen from Additional file 1: Fig. S12, even at concentrations as high as $1000 \mu \mathrm{g} / \mathrm{mL}$, the samples showed high viability over the entire concentration range. When MDA-MB-231 cancer cells were incubated with various concentrations of DCA-UiODTDP and DCA-UiO-DTDP-FA, the nanocomposites showed little toxicity to concentrations up to $500 \mu \mathrm{g} /$ $\mathrm{mL}$, and cell viability was about $80 \%$, even with the active targeting capability of DCA-UiO-DTDP-FA. 
Moreover, the incubation of DCA-UiO-DTDP and DCA-UiO-DTDP-FA with MCF-10A cells revealed that the DCA-UiO-DTDP-FA had no effect on normal cell proliferation in vitro. These data demonstrate that DCA-UiO-DTDP-FA shows excellent biocompatibility in vitro.

According to the report, DCA can enhance the cytotoxic activity of anticancer drugs such as 5 -fluorouracil (5-FU) and reducing the drug resistance of cancer cells. We selected 5-FU as a typical anticancer drug to evaluate drug loading and release behaviors and the enhanced cytotoxicity ability of 5-FU-DCA-UiO-DTDP-FA. DCAUiO-DTDP-FA and 5-FU were used for drug loading experiments in an excess of 5-FU in methanol, and the concentration of 5-FU was measured after various concentration ratios between nanoparticles and drug (see Additional file 1: Table S1). The UV/Vis absorbance of the solution was measured at regular interval to determine the amount of loading of 5-FU on the DCA-UiO-DTDPFA based on the UV/Vis absorbance and the standard absorbance curve of 5-FU in Additional file 1: Fig. S13. The results of the 5-FU loading experiments confirmed that the loading efficiency of DCA-UiO-DTDP-FA reached $31.6 \mathrm{wt} \%$ after $24 \mathrm{~h}$.

The release of 5-FU from the 5-FU-DCA-UiO-DTDPFA nanoparticles was investigated in the presence of dithiothreitol (DTT, a reducing agent that mimics the action of GSH, which is in the microenvironment of cancer cells provides a reducing environment.). It was assumed that the release of 5-FU from the nanoparticles occurs due to the breakage of disulfide bonds. DTDPA modified DCAUiO-66-NH2 nanoparticles can form disulfide bonds with organic ligands on the surface of the nanoparticles, and then coordinate with $\mathrm{NH}_{2}$-FA to limit drug leakage as a gate. GSH and its oxidized form (GSSG) are responsible for the formation of intracellular redox buffer [33]. Intracellular GSH attacks the thiolate moiety and is oxidized in the process as it cleaves the existing disulfide bonds. The encapsulation and release of the drug from the DCA-UiO-DTDP-FA were investigated at $37{ }^{\circ} \mathrm{C}$ with or without DTT. As shown in Fig. 3a, the release curve indicates that about $80 \%$ of 5 -FU was released from the nanoparticles after $24 \mathrm{~h}$ in the presence of $10 \mathrm{mM}$ DTT. This result was attributed to the higher number of disulfide bonds that were cleaved in the presence of high concentration of DTT. Compared to the control experiment without DTT, limited drug was released within $24 \mathrm{~h}$. The results showed that 5-FU was rapidly released from the nanoparticles in excess of DTT, mimicking the GSH present in cancer cells and the stability in plasma. The above results are of great significance to anticancer drug delivery systems based on post-synthetic modified stimuli-responsive MOFs nanoparticles.

Free 5-FU itself has significant dose-responsive cytotoxic behavior not only on cancer cells but also on normal cells (in Fig. 3b and Additional file 1: S14), but the cytotoxicity of the 5-FU-DCA-UiO-DTDP-FA samples showed higher toxicity to MDA-MB-231 cancer cells compared to 5-FU at same concentration in Fig. 3b. This may be the result of more efficient cell internalization of the nanoparticles with FA conjugation, or the DCA in the MOF structure had synergistic effect with 5-FU. To confirm this, we incubated the cells with 5-FU and NaDCA pure drugs mixed solution. Interestingly, the addition of DCA had enhanced the 5-FU effect, while the pure drug combination was still less efficiency comparing to
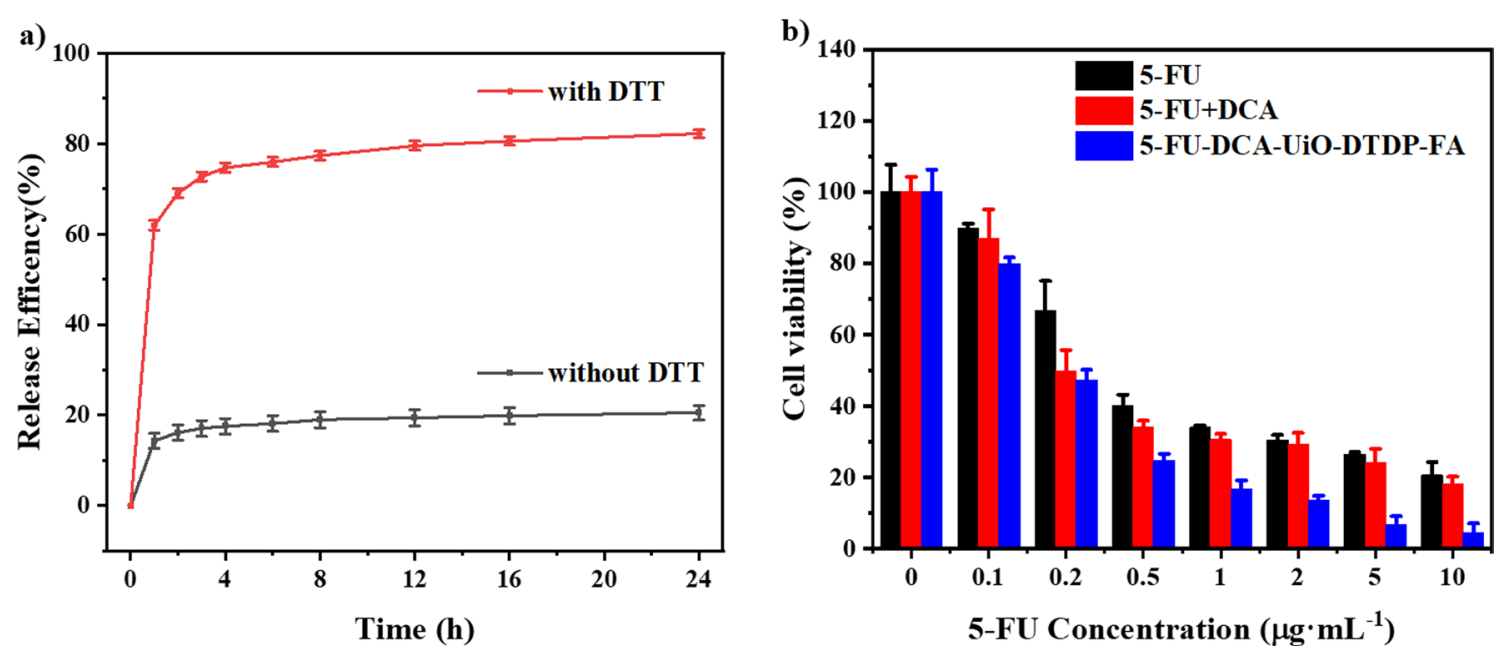

Fig. 3 The in vitro release of 5-FU from 5-FU-DCA-UiO-DTDP-FA (a), and viabilities of MDA-MB-231 cancer cells (b) cultured with 5-FU, 5-FU + DCA, and 5-FU-DCA-UiO-DTDP-FA 
the nanoparticles. 5-FU-DCA-UiO-DTDP-FA showed a greater apparent cytotoxic effect in all tested concentrations, in comparison to free 5-FU and free 5-FU + DCA. In the case of MCF-10A cell, as shown in Additional file 1: Fig. S14, the nanoparticles had limited cytotoxicity, which proved its selectivity towards cancer cells to normal cells.

To access the cellular uptake of DCA-UiO-DTDP-FA and DCA-UiO-DTDP, confocal laser scanning microscopy (CLSM) images were recorded for MDA-MB-231 cancer cells and MCF-10A cells incubated with the nanocomposites for 1,6 , and $16 \mathrm{~h}$ at $37{ }^{\circ} \mathrm{C}$ respectively, as shown in Figs. 4 and 6. For each figure, the cell nucleus is stained with $4^{\prime}, 6$-diamidino-2-phenylindole (DAPI), emits blue fluorescence, the DCA-UiO-DTDP-FA and DCA-UiO-DTDP was labeled with Sulfo-Cyanine5.5 NHS ester (Sulfo-Cy5.5) that has red fluorescence. In Fig. 4a, almost no red emission was observed in the first 1-6 h, which indicated that very few DCA-UiO-DTDPFA nanoparticles were phagocytized by MDA-MB-231 cancer cells. The same results were also shown in the DCA-UiO-DTDP nanoparticles group in Fig. 4b. However, when the incubation time was extended to $16 \mathrm{~h}$, compared with the same time in Fig. $4 \mathrm{~b}$, much stronger Sulfo-Cy5.5 red fluorescence emission was observed in the cytoplasm and cell nucleus in DCA-UiO-DTDP-FA group (Fig. 4a) comparing to DCA-UiO-DTDP group, which indicated that more $\mathrm{NH}_{2}$-FA-modified nanoparticles were uptaken by MDA-MB-231 cancer cells. The same results can also be seen from the mean fluorescent intensity (MFI) of the different cellular populations treated with different groups (Fig. 5). The MFI of the DCA-UiO-DTDP-FA group increased significantly, which was much higher than that of the DCA-UiODTDP group. Meanwhile, no significant red emission was observed during the entire uptaking process in MCF-10 cells (Fig. 6), which means that the two types of nanoparticles were indiscriminately consumed by normal cells and the passive uptake of normal cells was also less active than cancer cells. These results confirm that the prepared nanoparticles can be effectively phagocytized by MDAMB-231 cancer cells through receptor-mediated endocytosis, thereby achieving the tumor cells targeting.

\section{Conclusion}

In this work, by incorporating DCA at defect sites of UiO-66- $\mathrm{NH}_{2}$, we have produced a MOF with high drug loading capacity. Then, we carried out two post-synthetic modification steps to functionalize the MOF with redox stimuli-responsive and tumor targeting through anchoring functional FA molecules to the disulfide bond anhydrate modified surface of MOFs. By using the anticancer drug $5-\mathrm{FU}$ as the model drug, the drug showed redox responsive release, which is relevant to cancer

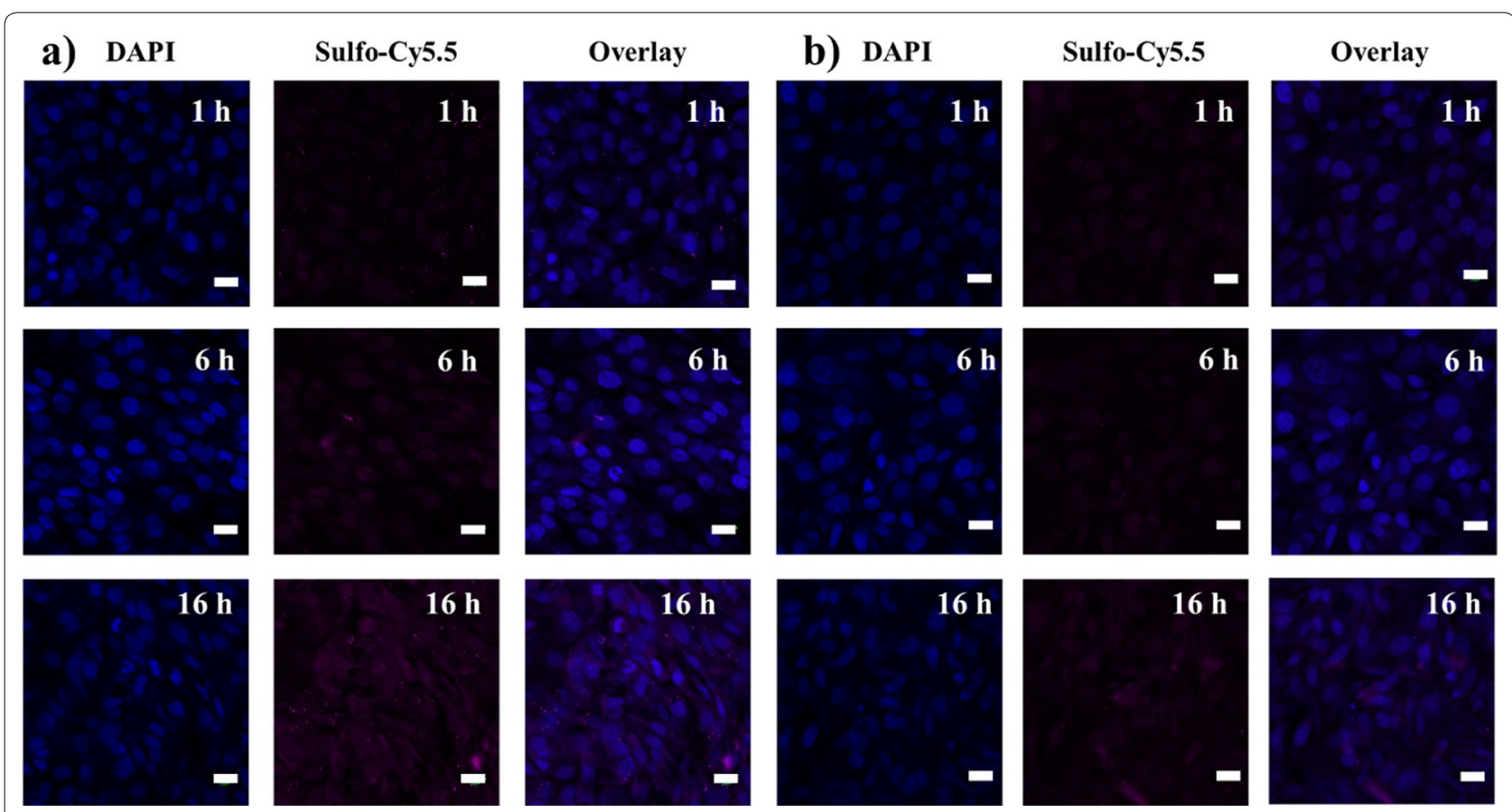

Fig. 4 Confocal laser scanning microscopy of MDA-MB-231 cancer cells incubated with a DCA-UiO-DTDP-FA + Sulfo-Cy5.5 and b DCA-UiO-DTDP + Sulfo-Cy5.5 for 1, 6, and $16 \mathrm{~h}$ at $37^{\circ} \mathrm{C}$. Each series shows cell nuclei (dyed blue with DAPI for visualization), DCA-UiO-DTDP-FA + Sulfo-Cy5.5 or DCA-UiO-DTDP + Sulfo-Cy5.5, and a merging of the two images. The scale bar in each image is $20 \mu \mathrm{m}$ 
a)

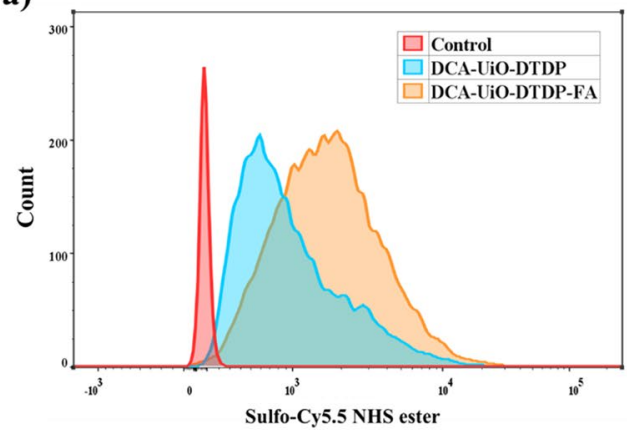

b)

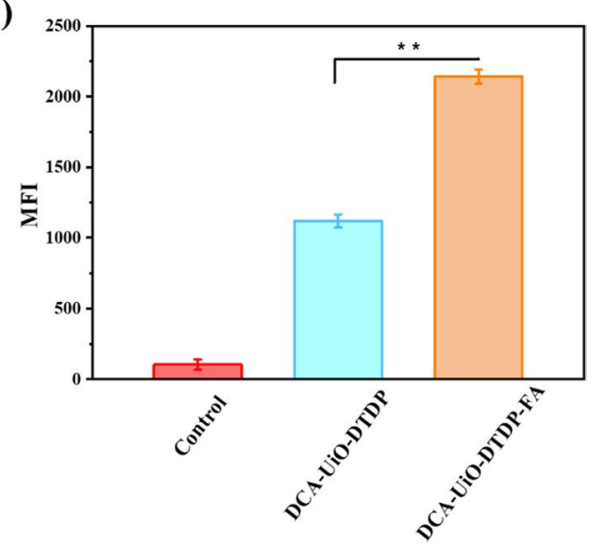

Fig. 5 a The flow cytometry analysis of cellular uptake of the control group, DCA-UiO-DTDP and DCA-UiO-DTDP-FA at $16 \mathrm{~h}$ for MDA-MB-231, and $\mathbf{b}$ corresponding mean fluorescent intensities with DCA-UiO-DTDP and DCA-UiO-DTDP-FA nanoparticles. The difference of tumor uptake in DCA-UiO-DTDP and DCA-UiO-DTDP-FA groups were statistically significant $(* * P<0.01)$

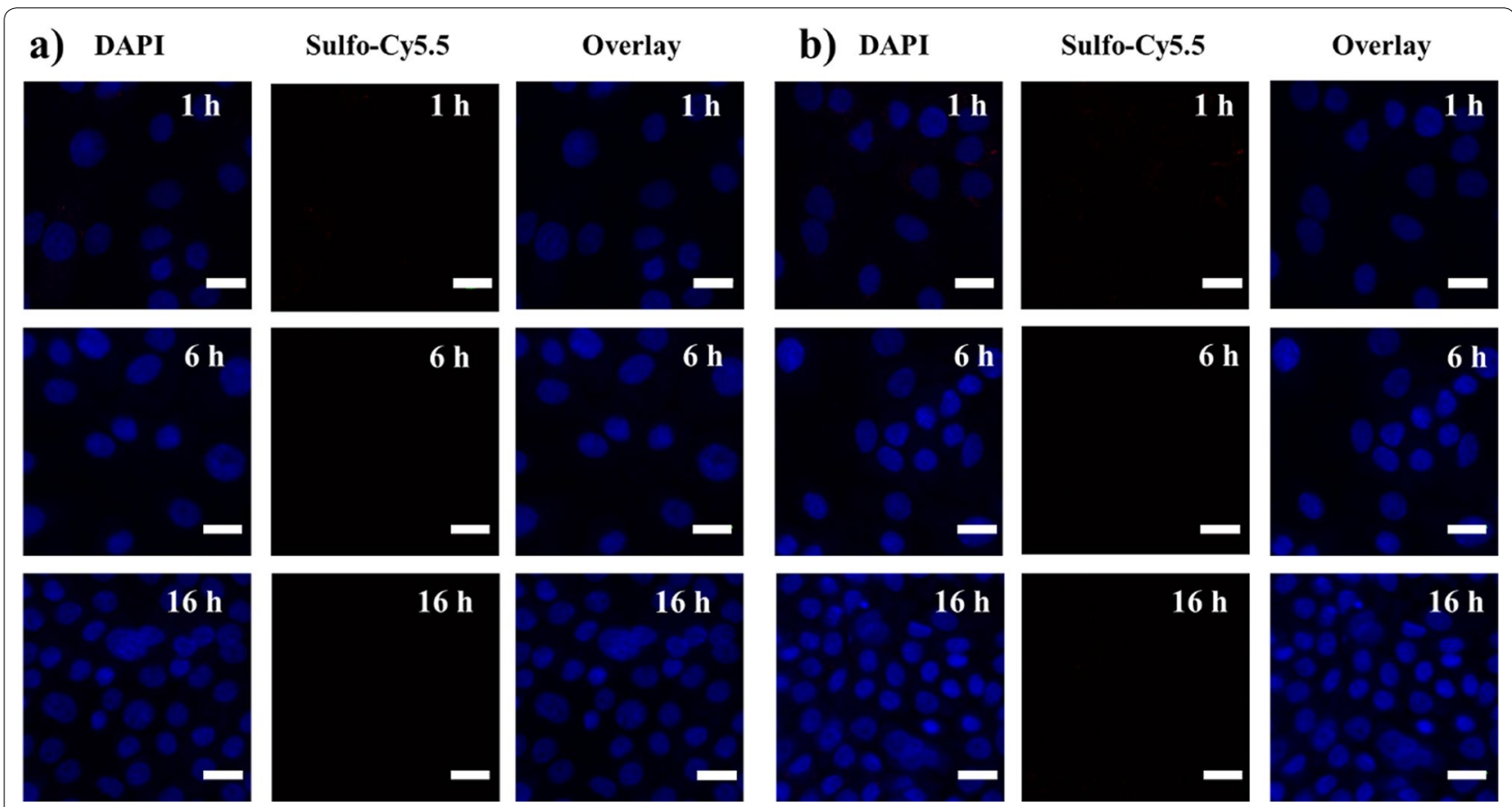

Fig. 6 Confocal laser scanning microscopy of MCF-10A cells incubated with a DCA-UiO-DTDP-FA+ Sulfo-Cy5.5 and b DCA-UiO-DTDP + Sulfo-Cy5.5 for 1,6 , and $16 \mathrm{~h}$ at $37^{\circ} \mathrm{C}$. Each series shows cell nuclei (dyed blue with DAPI for visualization), DCA-UiO-DTDP-FA + Sulfo-Cy5.5 or DCA-UiO-DTDP + Sulfo-Cy5.5, and a merging of the two images. The scale bar in each image is $20 \mu \mathrm{m}$

microenvironment. In addition, cell culture experiments have proved that the building block DCA in MOF structure can enhance the effect of 5-FU on cancer cell inhibition, moreover the 5-FU-DCA-UiO-DTDP-FA nanocomposite had enhanced cytotoxicity comparing to pure 5-FU+DCA mixture. More interestingly, the folic acid-modification on MOF had enhanced the drug uptake in cancer cells and the nanoparticles had clear selection towards cancer cells and they did not inhibit healthy cells. The results presented here indicate that MOFs are flexible in building blocks and surface functionalization, which had great potential in different types of tailored drug delivery systems. Delivery of multiple drugs from MOF-based platform has the potential to overcome issues with resistance and poor efficacy, the combination of targeting components with MOFs 
enhances tumor specificity for efficient targeted drugdelivery systems and has great potential for clinical applications.

\section{Experimental section}

\section{Materials and Instruments}

Unless otherwise stated, all the other chemicals and solvents were purchased for commercial use without further purification. $\mathrm{ZrCl}_{4}$ (anhydrous, powder, 99.99\% trace metals), 2-Aminoterephthalic acid $\left(\mathrm{H}_{2} \mathrm{~N}-\mathrm{H}_{2} \mathrm{BDC}\right.$, 99\%), Dichloroacetic acid (DCA, ReagentPlus ${ }^{\circledR}, \geq 99 \%$ ), 5-Fluorouracil (5-FU, $\geq 99 \%$, HPLC) and Sodium dichloroacetate (98\%) were obtained from Sigma-Aldrich. HPLC was performed on an Agilent 1100 HPLC (Agilent Technologies, USA) using a Waters Symmetry Shield RP18 Column $(4.6 \times 250 \mathrm{~mm}, 5 \mathrm{~mm}$, Waters Corporation, USA). Powder X-Ray Diffraction (PXRD) patterns were obtained on a Bruker D8 diffractometer using $\mathrm{Cu}$ $\mathrm{K} \alpha$ radiation with $40 \mathrm{~mA}$ and $40 \mathrm{kV}$, and the dates were collected within the $2 \theta$ range of $5^{\circ}-50^{\circ}$. Nuclear Magnetic Resonance Spectroscopy (NMR) were recorded on Bruker AMX 500 (1H-NMR at $500 \mathrm{MHz}$ ) spectrometers. UV-Vis Spectroscopy was recorded using a Thermo scientific Nanodrop 2000 spectrophotometer. Transmission electron microscopy (TEM) was performed on a JEOL JEM-1400Plus electron microscope operated at $80 \mathrm{kV}$. Fourier-transform Infrared Spectroscopy (FT-IR): FT-IR spectra of solid samples were collected using a PerkinElmer Spectrum Two FT-IR Spectrometer.

\section{Biological studies}

Cell proliferation assay Roche diagnostics reagent (WST1), phosphate-buffered saline (PBS), Penicillin-Streptomycin, and dimethylsulfoxide (DMSO) for cell culture solution were purchased from Sigma Aldrich. Dulbecco's modified eagle's medium (DMEM), trypsin-EDTA $(1 \mathrm{X})$, and fetal bovine serum (FBS) were purchased from Lonza. Minimum essential medium non-essential amino acids (MEM NEAA, 100X) was purchased from Gibco. L-Glutamine 100X was purchased from Biowest. Human breast cancer cell line MDA-MB-231 and normal breast cell line MCF-10A were used for in vitro studies. The MDA-MB-231 cells were cultured in highglucose DMEM containing 10\% heat-inactivated fetal bovine serum, $0.5 \%$ penicillin-streptomycin, $1 \%$ MEM NEAA, and $1 \%$ L-glutamine at $37^{\circ} \mathrm{C}$ in the sterile condition of $5 \% \mathrm{CO}_{2}$ incubator with the humidified atmosphere. MCF-10A were cultured in DMEM/F12 Medium (Gibco) containing 5\% FBS (Gibco), Glucose (Sigma, $4,5 \mathrm{~g} / \mathrm{L}$ ), human insulin (Sigma, $10 \mu \mathrm{g} / \mathrm{mL}$ ), hydrocortisone (Sigma, $0.5 \mu \mathrm{g} / \mathrm{mL}$ ), epidermal growth factor EGF (Peprotech, $20 \mathrm{ng} / \mathrm{mL}$ ) and $2 \mathrm{mM} \mathrm{L}$-glutamine (Gibco), in a humidified incubator with $5 \% \mathrm{CO}_{2}$. MDA-MB-231 cells are obtained from Prof. Jessica Rosenholm (Åbo Akademi, Turku, Finland) and MCF-10A cells are provided by Prof. Jukka Westermarck (Turku Bioscience Centre, Turku, Finland). Cells are exponentially cultured as monolayer up to $70-80 \%$ confluency. After optimal growth, cells were detached using trypsin-EDTA. All the cell culture work was performed under sterile conditions.

\section{Synthesis of DCA-UiO-66- $\mathrm{NH}_{2}$}

Following these general steps to prepare DCA-UiO66- $\mathrm{NH}_{2}$ nanoparticles: in separate vessels, $\mathrm{ZrCl}_{4}(1.0 \mathrm{~g}$, $4.2 \mathrm{mmol})$ and $\mathrm{H}_{2} \mathrm{BDC}-\mathrm{NH}_{2}(761.0 \mathrm{mg}, 4.2 \mathrm{mmol}$ ) were dissolved in $150 \mathrm{~mL}$ of DMF. After mixing both precursor solutions transferring into a $250 \mathrm{~mL}$ vial, dichloroacetic acid (DCA, $3.0 \mathrm{~mL}, 36.0 \mathrm{mmol}$ ) was added to the reaction mixture, after gently stirring, it was placed in the oven at $120{ }^{\circ} \mathrm{C}$ for $24 \mathrm{~h}$. After cooling to room temperature, the powders were collected by centrifugation and washed 3 times with DMF and 3 times with ethanol by dispersion centrifugation cycles. The resulting MOFs were dried under vacuum for at least $12 \mathrm{~h}$ before analysis.

\section{Synthesis of 3,3'-dithiodipropionic anhydride (DTDPA)}

$1.0 \mathrm{~g}$ of $3,3^{\prime}$-dithiodipropionic acid (DTDP) was added to $5.0 \mathrm{~mL}$ of acetyl chloride and refluxed at $65{ }^{\circ} \mathrm{C}$ for $2 \mathrm{~h}$. The resulting solution was cooled to room temperature and the solvent was evaporated. The residue was then precipitated in diethyl ether and washed repeatedly with diethyl ether. The product 3,3'-dithiodipropionic anhydride (DTDPA) was dried in a vacuum desiccator overnight and used directly in the next step.

\section{Postsynthetic modification of DCA-UiO-66- $\mathrm{NH}_{2}$ by using DTDPA (as DCA-UiO-DTDP)}

The amine functional groups of the MOF reacted with DTDPA to form carboxylic acid-functionalized DCA-UiO-DTDP. In a typical post-synthetic modification reaction, after synthesizing of DCA-UiO-66- $\mathrm{NH}_{2}$ (ca. $4.0 \mathrm{mmol}$ of $-\mathrm{NH}_{2}$ ), about $4.0 \mathrm{mmol}$ of DTDPA $(192.25 \mathrm{mg})$ was placed in the above DMF solution. After the sample was left at room temperature for $24 \mathrm{~h}$, the solution was decanted, and the crystals were washed with fresh DMF and ethanol $(3 \times 5 \mathrm{~mL})$ and then dried under vacuum.

\section{Synthesis of aminated folic acid}

For amination, folic acid $(1.0 \mathrm{mmol})$ dissolved in $30.0 \mathrm{ml}$ DMSO with 1-Ethyl-3-(3-dimethylaminopropyl)carbodiimide (EDC, $1.2 \mathrm{mmol}$ ) and $\mathrm{N}$-Hydroxysuccinimide (NHS, $2 \mathrm{mmol}$ ) was reacted at $50^{\circ} \mathrm{C}$ for $6 \mathrm{~h}$. The obtained folic acid-NHS was mixed with ethylenediamine $(10.0 \mathrm{mmol})$ and $500.0 \mu \mathrm{g}$ pyridine and allowed to react at room temperature overnight. The crude product was 
precipitated by adding an excess of acetonitrile, filtered and washed three times with diethyl ether, and then dried under vacuum.

\section{Preparation of 5-FU-loaded DCA-UiO-DTDP}

Fresh DCA-UiO-DTDP was immersed in methanol for $24 \mathrm{~h}$, and then the extract was discarded. Fresh methanol was then added, and the sample was immersed for another $24 \mathrm{~h}$ to remove $\mathrm{H}_{2} \mathrm{O}$ and DMF. The sample was then treated with dichloromethane to remove methanol solvates using the same procedures. After dichloromethane was removed by decantation, the sample was activated by using a dynamic vacuum at $100{ }^{\circ} \mathrm{C}$ for $12 \mathrm{~h}$. To load 5-FU into the pores of DCA-UiO-DTDP, dehydrated DCA-UiO-DTDP (5 mg) was dispersed in a 5- FU containing $\mathrm{MeOH}$ solution $(5 \mathrm{~mL})$ and stirred for 1 day to yield a uniform light-yellow solution. The 5-FU-DCAUiO-DTDP was collected by centrifugation $(13,000 \mathrm{rpm}$ for $10 \mathrm{~min})$ and washed with $\mathrm{MeOH}(\times 2)$ by dispersive centrifugation cycles to ensure that no residual 5-FU remained on the particle surfaces. The resulting MOFs were dried under vacuum at least $24 \mathrm{~h}$ before analysis. The amount of 5-FU adsorption in the porous solids was estimated by UV-Vis. The wavelength used for 5-FU quantification was $266 \mathrm{~nm}$.

\section{Fabrication of 5-FU-DCA-UiO-DTDP-FA}

Same as encapsulating 5-FU in DCA-UiO-DTDP, dehydrated DCA-UiO-DTDP ( $5 \mathrm{mg}$ ) was dispersed in a 5- FU $\mathrm{MeOH}$ solution $(3 \mathrm{mg} / \mathrm{mL}, 5 \mathrm{~mL})$ and stirred for 1 day to yield a homogenous light-yellow solution. Then according to the ration 1:1.2:2, EDC and NHS were added to activate the carboxyl groups of DCA-UiO-DTDP. After $6 \mathrm{~h}, 5 \mathrm{mg}$ of aminated FA was added to the solution. 5-FU-DCA-UiO-DTDP-FA were collected by centrifugation $(12,000 \mathrm{r} / \mathrm{min}$ for $20 \mathrm{~min})$ and washed with $\mathrm{MeOH}$ (x2) by dispersive centrifugation cycles to ensure that there is no residual 5-FU on the particle surfaces. The resulting MOFs were dried under vacuum at least $24 \mathrm{~h}$ before analysis. The amount of 5-FU adsorption in the porous solids was estimated by UV-Vis. The wavelengths used for 5-FU quantification was $266 \mathrm{~nm}$.

\section{In vitro drug release kinetics}

The cumulative levels of 5 -FU released from 5-FUDCA-UiO-DTDP-FA nanoparticles were characterized using the dialysis method. Briefly, $15 \mathrm{mg}$ of drug-loaded 5-FU-DCA-UiO-DTDP-FA was dispersed in $1.5 \mathrm{~mL}$ of PBS buffer solution (pH 7.4), and placed in a dialysis bag $(\mathrm{MWCO}=500 \mathrm{D})$, and then immersed in $50 \mathrm{~mL}$ of PBS with or without DTT $(10 \mathrm{mM})$, and resuspended in a $37{ }^{\circ} \mathrm{C}$ constant temperature water bath with horizontal shaking. Here, DTT was used as a reducing agent that mimics the action of GSH, which provides a reducing environment in the microenvironment of cancer cells. At each time interval, $1 \mathrm{~mL}$ of the incubation solution was taken out and replenished with an equal volume of corresponding PBS. The content of 5-FU in the removed sample was monitored by HPLC, where the detection wavelength was $265 \mathrm{~nm}$. The flow rate of the mobile phase was $1.0 \mathrm{~mL} / \mathrm{min}$, and the temperature was set at $30{ }^{\circ} \mathrm{C}$.

\section{In vitro cellular assay Cell viability assay}

5-FU acts as a thymidylate synthase (TS) inhibitor, so it needs to reach the nucleus of cancer cells to be effective, while DCA inhibits pyruvate kinase and therefore acts on mitochondria. The cytotoxicity of the free DCA, 5-FU, 5-FU+DCA, DCA-UiO-DTDP, DCA-UiO-DTDP-FA, and 5-FU-DCA-UiO-DTDP-FA were measured against breast cancer cell line MDA-MB-231 and non-tumorigenic epithelial cell line MCF-10A, using the WST-1 cell proliferation assay Roche diagnostics reduction assay.

First, breast cancer cell line MDA-MB-231 and nontumorigenic epithelial cell line MCF-10A were cultivated in a 96-well plate, with 4000-5000 cells per well, and incubated for $24 \mathrm{~h}$ with $\mathrm{CO} 2\left(5 \%, 37^{\circ} \mathrm{C}\right)$. Thereafter, the DCA suspension was diluted to concentrations of $0.1,0.2,0.5,1.0,2.0,5.0$ and $10.0 \mathrm{mg} / \mathrm{mL}$. 5 -FU suspension was diluted to concentrations of $0.1,0.2,0.5$, 1.0, 2.0, 5.0 and $10.0 \mu \mathrm{g} / \mathrm{mL}$. 5-FU+DCA suspensions based on the concentrations of 5-FU and the ratio of DCA:5-FU in the final formulation was diluted to 0.1, 0.2, $0.5,1.0,2.0,5.0$ and $10.0 \mu \mathrm{g} / \mathrm{mL}$. DCA-UiO-DTDP and DCA-UiO-DTDP-FA suspensions were diluted to concentrations of 10, 20, 50, 100, 200, 500 and $1000 \mu \mathrm{g} / \mathrm{mL}$. 5-FU-DCA-UiO-DTDP-FA suspensions based on 5-FU concentrations in the final formulation was diluted to 0.1 , $0.2,0.5,1.0,2.0,5.0$ and $10.0 \mu \mathrm{g} / \mathrm{mL}$. Then the solutions were added to the wells and incubated for another $24 \mathrm{~h}$. As blank controls, only eight wells were left with culture, and then DMSO $(10.0 \mu \mathrm{L})$ was added to each well. Subsequently, WST-1 cell proliferation assay solution $(10.0 \mu \mathrm{L})$ was added to each well, and then incubated at $37{ }^{\circ} \mathrm{C}$ for another $2 \mathrm{~h}$. Finally, the absorbances of the solutions at $490 \mathrm{~nm}$ were measured by using a microplate reader.

\section{Detection of cellular uptake and kinetics using confocal analysis}

Cellular uptake: The cellular uptake of breast cancer cell line MDA-MB-231 and non-tumorigenic epithelial cell line MCF-10A were measured by confocal laser scanning microscopy (CLSM). Generally, MDA-MB-231 and MCF-10A cells $\left(2 \times 10^{5}\right.$ per well $)$ were first seeded in confocal dishes and grown for $12 \mathrm{~h}$ before the uptake 
study, and then incubated with the prepared DCA-UiODTDP and DCA-UiO-DTDP-FA suspensions $(2 \mathrm{~mL}$, $500 \mathrm{mg} / \mathrm{mL})$ at $37^{\circ} \mathrm{C}$ for different times $(1,6$ and $16 \mathrm{~h})$. At different time points, the cells were washed three times with PBS. Thereafter, the cells were fixed with $4 \%$ paraformaldehyde $(2 \mathrm{~mL} /$ well $)$ at $37{ }^{\circ} \mathrm{C}$ for $10 \mathrm{~min}$ and further rinsed three times with PBS. For nucleus labeling, the nucleus were stained with DAPI solution $(20 \mathrm{mg} /$ $\mathrm{mL}$ in PBS, $1 \mathrm{~mL} /$ well) for $5 \mathrm{~min}$, and the cells were then washed three times with PBS again, and the samples were examined with a Zeiss LSM880 with air scan instrument.

\section{Flow cytometric detection of cellular uptake and kinetics}

The cellular uptake kinetics was studied using flow cytometer. $1-5 \times 10^{5}$ density of breast cancer cell line MDA-MB-231 and non-tumorigenic epithelial cell line MCF-10A were detached using trypsin and resuspended in $2.00 \mathrm{~mL}$ cell culture serum-free media containing DCA-UiO-DTDP and DCA-UiO-DTDP-FA at the concentration $500 \mathrm{mg} / \mathrm{mL}$ at different periods up to $24 \mathrm{~h}$. After incubation at $37{ }^{\circ} \mathrm{C}$ cells suspension was diluted with $2 \mathrm{~mL}$ of PBS and centrifuged at $800 \times \mathrm{g}$. The cell pellet was washed twice with PBS by centrifugation and resuspended in $500 \mu \mathrm{L}$ of serum-free media. Fluorescence analysis was performed with BD LSRFORTESSA using Alexa Fluor 700-A channels and a $640 \mathrm{~nm}$ emission filter.

\section{Supplementary information}

Supplementary information accompanies this paper at https://doi. org/10.1186/s42833-020-00013-y.

Additional file 1. Fig. S1. Schematic of DCA-UiO-66- $\mathrm{NH}_{2}$ with DCA capping $\mathrm{Zr}_{6}$ clusters to form defects; Fig. S2. Comparison of FTIR spectra of DCA-loaded UiO-66- $\mathrm{NH}_{2}$ with the empty UiO-66- $\mathrm{NH}_{2}$ and DCA; Fig. S3. The post-synthetic modification of DCA-UiO-66- $\mathrm{NH}_{2}$ with 3,3'-dithiodipropionic anhydrides; Fig. S4. The synthesis procedure of DTDPA from DTDP; Fig. S5. 1 H NMR spectra of DTDP and DTDPA; Fig. S6. 1H NMR spectra of DCA-UiO-66-NH ${ }_{2}$; Fig. S7. ' $1 \mathrm{H}$ NMR spectra of DCA-UiO-66-DTDP; Fig. S8. The synthesis of aminated folic acid; Fig. $\mathbf{5 9}{ }^{1}$ H NMR spectra of aminated folic acid; Fig. S10. Comparison of the FTIR spectra of the DCA-UiO66-DTDP-FA and DCA-UiO-66-DTDP and FA-NH 2 ; Fig. S11. Cells viability of MDA-MB-231 and MCF-10A cells incubated with different concentrations of NaDCA for 72 h; Fig. S12. Cells viabilities of MDA-MB-231 cancer cell and MCF-10A cells cultured with different concentrations of DCA-UiODTDP and DCA-UiO-DTDP-FA nanoparticles for $72 \mathrm{~h}$; Table S1. Determination of the 5-Fluorouracil loading efficiency; Fig. S13. The standard curve of 5-FU in $\mathrm{MeOH}$; Fig. S14. The viabilities of MCF-10A normal cells cultured with 5-FU, 5-FU+DCA, and 5-FU-DCA-UiO-DTDP-FA.

\section{Abbreviations}

MOFs: metal-organic frameworks; PSM: post-synthetic modification; TEM: transmission electron microscopy; FT-IR: Fourier-transform infrared spectroscopy; PXRD: powder X-ray diffraction; NMR: nuclear magnetic resonance spectroscopy; CLSM: confocal laser scanning microscopy; MFI: mean fluorescent intensity; FA: folic acid; DCA: dichloroacetic acid; 5-FU: 5-fluorouracil; DTDPA: 3,3'-dithiodipropionic acid anhydride.

\section{Acknowledgements}

The authors acknowledge Markus Peurla (Institute of Biomedicine, University of Turku) for providing useful advice TEM measurements.

\section{Authors' contributions}

$\mathrm{CL}$ and $\mathrm{XX}$ performed data processing and wrote and revised the manuscript. $\mathrm{CL}, \mathrm{JY}$, and JZ performed experiments and provided the raw data. JZ, DW and $\mathrm{HZ}$ were the Pls. $\mathrm{CL}$ and $\mathrm{HZ}$ provided strategic guidance during development of idea, wrote and revised the manuscript. All authors read and approved the final manuscript.

\section{Funding}

This work was supported by Distinguished Clinical Investigator Grant of Jiangsu Province, China (Grant No. JSTP201701), Jiangsu Provincial Key Research and Development Programme (Grant No. BE2018690), Academy of Finland (Grant No. 328933) and Sigrid Jusélius Foundation (Decision No. 28002247K1). The funder had no role in study design, data collection and analysis, decision to publish, or preparation of the manuscript.

\section{Availability of data and materials}

The datasets generated during and/or analyzed during the current study are available from the corresponding author on reasonable request.

\section{Ethics approval and consent to participate}

Not applicable.

\section{Consent for publication}

Not applicable.

\section{Competing interests}

The authors declare that they have no competing interests.

\section{Author details \\ ${ }_{1}^{1}$ Pharmaceutical Sciences Laboratory and Turku Bioscience Center, Åbo Akademi University, 20520 Turku, Finland. ${ }^{2}$ Experimental Hematology and Biochemistry Lab, Beijing Institute of Radiation Medicine, Beijing 100850, People's Republic of China. ${ }^{3}$ Guangdong Pharmaceutical University, Guang- zhou 510006, People's Republic of China. ${ }^{4}$ Department of Radiology, Affiliated Hospital of Jiangsu University, Jiangsu University, Zhenjiang 212001, People's Republic of China.}

Received: 14 May 2020 Accepted: 9 June 2020

Published online: 02 July 2020

\section{References}

1. Patra JK, Das G, Fraceto LF, Campos EVR, Rodriguez-Torres MDP, AcostaTorres LS, et al. Nano based drug delivery systems: recent developments and future prospects. J Nanobiotechnol. 2018;16:71.

2. Su J, Chen F, Cryns VL, Messersmith PB. Catechol polymers for pHresponsive, targeted drug delivery to cancer cells. J Am Chem Soc. 2011:133:11850-3.

3. Shim MS, Kwon YJ. Stimuli-responsive polymers and nanomaterials for gene delivery and imaging applications. Adv Drug Deliv Rev. 2012;64:1046-58

4. Zhang YF, Huang YX, Li S. Polymeric micelles: nanocarriers for cancertargeted drug delivery. AAPS PharmSciTech. 2014;15:862-71.

5. Dong YZ, Eltoukhy AA, Alabi CA, Khan OF, Veiseh O, Dorkin JR, et al. Lipid-like nanomaterials for simultaneous gene expression and silencing in vivo. Adv Healthc Mater. 2014;3:1392-7.

6. Gu FX, Karnik R, Wang AZ, Alexis F, Levy-Nissenbaum E, Hong S, et al. Targeted nanoparticles for cancer therapy. Nano Today. 2007;2:14-21.

7. Aryal S, Grailer JJ, Pilla S, Steeber DA, Gong SQ. Doxorubicin conjugated gold nanoparticles as water-soluble and $\mathrm{pH}$-responsive anticancer drug nanocarriers. J Mater Chem. 2009;19:7879-84.

8. Xu XY, Ho W, Zhang XQ, Bertrand N, Farokhzad O. Cancer nanomedicine: from targeted delivery to combination therapy. Trends Mol Med. 2015;21:223-32. 
9. Li ZG, Liu J, Hu Y, Howard KA, Li Z, Fan XL, et al. Multimodal imagingguided antitumor photothermal therapy and drug delivery using bismuth selenide spherical sponge. ACS Nano. 2016;10:9646-58.

10. Mura S, Nicolas J, Couvreur P. Stimuli-responsive nanocarriers for drug delivery. Nat Mater. 2013;12:991-1003.

11. Ren JT, Hu YW, Lu CH, Guo WW, Aleman-Garcia MA, Ricci F, et al. pHresponsive and switchable triplex-based DNA hydrogels. Chem Sci. 2015;6:4190-5.

12. Cheng EJ, Xing YZ, Chen P, Yang Y, Sun YW, Zhou DJ, et al. A pH-triggered, fast-responding DNA hydrogel. Angew Chem Int Ed. 2009;48:7660-3.

13. Qiao Y, Ping Y, Zhang HB, Zhou B, Liu FY, Yu YH, et al. Laser-activatable cus nanodots to treat multidrug-resistant bacteria and release copper ion to accelerate healing of infected chronic nonhealing wounds. ACS Appl Mater Interfaces. 2019;11:3809-22.

14. Takashima Y, Hatanaka S, Otsubo M, Nakahata M, Kakuta T, Hashidzume $A$, et al. Expansion-contraction of photoresponsive artificial muscle regulated by host-guest interactions. Nat Commun. 2012;3:1-8.

15. Ma XD, Ozliseli E, Zhang YZ, Pan GQ, Wang DQ, Zhang HB. Fabrication of redox-responsive doxorubicin and paclitaxel prodrug nanoparticles with microfluidics for selective cancer therapy. Biomater Sci. 2019;7:634-44.

16. Zhao ZH, Huang DT, Yin ZY, Chi XQ, Wang XM, Gao JH. Magnetite nanoparticles as smart carriers to manipulate the cytotoxicity of anticancer drugs: magnetic control and $\mathrm{pH}$-responsive release. J Mater Chem. 2012;22:15717-25.

17. Yu SF, Wu GL, Gu X, Wang JJ, Wang YN, Gao H, et al. Magnetic and $\mathrm{pH}$-sensitive nanoparticles for antitumor drug delivery. Colloid Surf B. 2013:103:15-22

18. Lai JP, Shah BR, Zhang YX, Yang LT, Lee KB. Real-time monitoring of ATP-responsive drug release using mesoporous-silica-coated multicolor upconversion nanoparticles. ACS Nano. 2015;9:5234-45.

19. Furukawa H, Gandara F, Zhang YB, Jiang JC, Queen WL, Hudson MR, et al. Water adsorption in porous metal-organic frameworks and related materials. J Am Chem Soc. 2014;136:4369-81.

20. Rimoldi M, Howarth AJ, DeStefano MR, Lin L, Goswami S, Li P, et al. Catalytic zirconium/hafnium-based metal-organic frameworks. ACS Catal. 2017;7:997-1014

21. Masih D, Chernikova V, Shekhah O, Eddaoudi M, Mohammed OF. Zeolitelike metal-organic framework(MOF) encaged Pt(II)-porphyrin for anionselective sensing. ACS Appl Mater Interfaces. 2018;10:11399-405.
22. Shimizu GKH, Taylor JM, Kim S. Proton conduction with metal-organic frameworks. Science. 2013;341:354-5.

23. Horcajada P, Serre C, Maurin G, Ramsahye NA, Balas F, Vallet-Regi M, et al. Flexible porous metal-organic frameworks for a controlled drug delivery. J Am Chem Soc. 2008;130:6774-80.

24. Horcajada P, Gref R, Baati T, Allan PK, Maurin G, Couvreur P, et al. Metalorganic frameworks in biomedicine. Chem Rev. 2012;112:1232-68.

25. Della Rocca J, Liu DM, Lin WB. Nanoscale metal-organic frameworks for biomedical imaging and drug delivery. Accounts Chem Res. 2011:44:957-68.

26. Wang ZQ, Cohen SM. Postsynthetic modification of metal-organic frameworks. Chem Soc Rev. 2009;38:1315-29.

27. Gamcsik MP, Kasibhatla MS, Teeter SD, Colvin OM. Glutathione levels in human tumors. Biomarkers. 2012;17:671-91.

28. Kim H, Jo A, Baek S, Lim D, Park SY, Cho SK, et al. Synergistically enhanced selective intracellular uptake of anticancer drug carrier comprising folic acid-conjugated hydrogels containing magnetite nanoparticles. Sci Rep. 2017:7:1-10.

29. Kandiah M, Nilsen MH, Usseglio S, Jakobsen S, Olsbye U, Tilset M, et al. Synthesis and stability of tagged UiO-66Zr-MOFs. Chem Mater. 2010;22:6632-40

30. Heshe D, Hoogestraat S, Brauckmann C, Karst U, Boos J, Lanvers-Kaminsky C. Dichloroacetate metabolically targeted therapy defeats cytotoxicity of standard anticancer drugs. Cancer Chemother Pharm. 2011;67:647-55.

31. Liu CY, Yuan J, Luo XM, Chen MH, Chen ZJ, Zhao YC, et al. Folate-decorated and reduction-sensitive micelles assembled from amphiphilic polymer-camptothecin conjugates for intracellular drug delivery. Mol Pharm. 2014;11:4258-69.

32. Lee ES, Na K, Bae YH. Polymeric micelle for tumor $\mathrm{pH}$ and folate-mediated targeting. J Control Release. 2003;91:103-13.

33. Saito G, Swanson JA, Lee KD. Drug delivery strategy utilizing conjugation via reversible disulfide linkages: role and site of cellular reducing activities. Adv Drug Deliv Rev. 2003;55:199-215.

\section{Publisher's Note}

Springer Nature remains neutral with regard to jurisdictional claims in published maps and institutional affiliations.
Ready to submit your research? Choose BMC and benefit from:

- fast, convenient online submission

- thorough peer review by experienced researchers in your field

- rapid publication on acceptance

- support for research data, including large and complex data types

- gold Open Access which fosters wider collaboration and increased citations

- maximum visibility for your research: over $100 \mathrm{M}$ website views per year

At BMC, research is always in progress.

Learn more biomedcentral.com/submissions 